\title{
TIDAL FORCE OF BLACK HOLES
}

\section{Arun Kenath \& Samartha C.A.*}

[The work presented in this paper is part of our project titled "Aspects of Black Hole Energetics" during the fourth semester of M.Sc. (Physics) under the guidance of Prof. C. Sivaram, IIA. We are very thankful to Prof. C. Sivaram for his guidance throughout the project and also to Prof. K. A. Chandrashekar, H.O.D, P.G Dept of Physics, Christ College, for the support and encouragement. ]

\section{Tidal Force}

The tidal force is a secondary effect of the force of gravity. It arises because the gravitational field is not constant across a body's diameter. When a body is acted on by the gravity of another body, the gravitational acceleration can vary significantly between the near side and the far side. This tends to distort the shape of the body without altering its volume.

Suppose that the gravitational field is due to one other body. Linearising Newton's law of gravitation around the centre of the reference body yields an approximate inverse cube law. Along the axis through the centres of the two bodies, this takes the form

$$
F_{1}-\frac{2 G M m r}{R^{3}}
$$

where $G$ is the gravitational constant, $M$ is the mass of the body producing the field, $m$ is the mass on which the tidal force octs, $R$ is the distance between the two

* PG Department of Physics, Christ College, Bangalore. 
bodies and $r<<R$ is the distance from the reference body's centre along the axis. This tidal force acts outwards both at the near side and at the far side of the body, leading to a bulge on both sides.

Tidal effects become particularly pronounced near small bodies of high mass, such as neutron stars or black holes, where they are responsible for the stretching and the eventual break up of the in falling matter.

In the case of a black hole, there is no surface to halt the fall. As things fall into a black hole the tidal forces become stronger and stronger until nothing can resist them. The bodies are stretched into thin streams of matter. Eventually, close to the singularity, they become large enough to tear molecules apart.

For a very large black hole such as those found at the centre of galaxies, this point will lie well inside the event horizon, so the object may cross the event horizon without any pulling whatsoever. For small black holes whose Schwarzschild radius is much closer to the singularity, the tidal effects may become large long before the object even reaches the event horizon [3].

Tidal forces will break up ordinary stars as they spiral in toward the engine and smear out the energy due to gravitational radiation. However, the galactic core will also contain compact objects like white dwarts, neutron stars and stellar mass black holes, the final evolutionary stages of stars. These compact objects will not break up when captured, and can emit detectable amounts of gravitational radiation.

Compact objects that pass near the central black hole will radiate gravitational waves as they swing around the central black hole, thereby progressively losing orbital energy and slipping toward the black hole's horizon. Compact objects on orbits such as these are rapidly removed from the cusp. These scattering events may save a compact object from capture by the central engine by changing the small compact object's angular momentum. This is more likely for smaller objects like white dwarfs and neutron stars. However, this same process will also scatter compact objects that were not in capture orbits, that is, were not passing close enough to the massive black hole, into capture orbits, and thereby ensuring a continual supply of compact objects for consumption by the central engine. Stellar mass black holes may persist and radiate strongly for several years in these capture orbits.

The orbits of these compact objects around the central engine will be highly relativistic. A stellar mass black hole may be going as fast as half the speed of light at its point of nearest approach to the massive black hole. These orbits will make very complicated waveforms, dependent on many parameters of the binary system [5]. 


\section{Tidal break up of a Star}

Consider a star of mass $M_{\text {stor }}$ and radius $R_{\text {stor }}$ approaching a black hole of mass $M_{\mathrm{BH}}$. The tidal force experienced at a point on the star closest to the black hole is given by, $\frac{G M_{B H} M_{\text {slor }}}{\left(D-R_{\text {slar }}\right)^{2}}$ And the force at a point farthest to the black hole is given by, $\frac{G M_{\text {gH }} M_{\text {slor }}}{\left(D-R_{\text {star }}\right)^{2}}$

Here $D$ is the distance from the centre of the star to the black hole. For the tidal break up of the star, the self-gravitational force of the star given by $\frac{G M_{\text {star }}}{R_{\text {stor }}^{2}}$ should be less than the tidal force exerted by the black hole on the star. That is,

$$
\frac{G M_{\text {stor }}}{R_{\text {stor }}^{2}} \leq \frac{G M_{B H} M_{\text {stor }}}{\left(D-R_{\text {stor }}\right)^{2}}-\frac{G M_{B H} M_{\text {slor }}}{\left(D+R_{\text {star }}\right)^{2}}
$$

From this we can determine the distance, at which the star will undergo the tidal break up as,

$$
D=R_{\text {star }}\left(4 \frac{M_{B H}}{M_{\text {star }}}\right)^{1 / 3}
$$

For a supermassive black hole of mass $10^{9} \mathrm{M}_{\Theta}$ and a star with mass and radius as that of the sun, this distance is $D \approx 10^{13} \mathrm{~m}$.

For the $10^{9} \mathrm{M}$ black hole, the Schwarzschild radius is of the order of $10^{12} \mathrm{~m}$. This implies that the distance for the tidal break up is, $D=10 R_{s}$.

The energy released during the tidal break up is equivalent to the gravitational binding energy [2] of the star. That is,

$$
\text { Energy released }=B E=\frac{3}{5} \frac{G M_{\text {stor }}^{2}}{R_{\text {stor }}}
$$


For a star like our sun this works out to about $10^{41} \mathrm{~J}$. This much of energy is released during the tidal break up of a star in the vicinity of a black hole.

This is one of the possible fuels for the central black holes at the centres of galaxies; such that it emits energies of the order of $10^{54} \mathrm{~J}$.

In the following fable we have illustrated the variation of the distance for the tidal break-up of a star like that of our sun with respect to the mass of the central black hole.

Table 1.1

\begin{tabular}{|c|c|c|}
\hline$M_{B H}\left(\right.$ in $\left.M_{Q}\right)$ & $D$ (in meters) & $D\left(\right.$ in $\left.R_{s}\right)$ \\
\hline $10^{9}$ & $10^{13}$ & 10 \\
$10^{6}$ & $10^{12}$ & $10^{3}$ \\
$10^{3}$ & $10^{11}$ & $10^{5}$ \\
\hline
\end{tabular}

\section{Tidal Break up of Compact Objects}

As mentioned earlier in the chapter, the compact objects like white dwarfs and neutron stars, do not undergo tidal break up in the vicinity of super massive black holes. In this section we will consider the mass of the black hole required to force the tidal break up of these compact objects.

In the case of white dwarfs, the electron degeneracy pressure balances the gravitational pressure.

Electron degeneracy pressure occurs when electrons are compressed into a very small volume. Since their positions are well known, they gain a large momentum in accordance with Heisenberg's uncertainty principle. This momentum creates a pressure, which is temperature-independent and is responsible for the stability of white dwarts.

It has a magnitude given as follows with usual notations,

$$
P=\frac{\pi^{2} \hbar^{2}}{5 m_{\mathrm{e}} m_{H}^{5 / 3}}\left(\frac{3}{\pi}\right)^{2 / 3}\left(\frac{\rho}{\mu_{\mathrm{e}}}\right)^{5 / 3}
$$


By equating this electronic degeneracy pressure with the gravitational pressure, we can determine the radius of the white dwarf [3].

$$
\frac{G M_{W D}^{2}}{R_{W D}^{4}}=\frac{\hbar^{2} M_{W D}^{5 / 3}}{m_{P}^{5 / 3} m_{e} R_{W D}^{5}}
$$

From this the radius of the white dwarf is obtained as $\approx 10^{6} \mathrm{~m}$

We have already obtained the distance for the tidal break up as

$$
D=R_{\text {stor }}\left(4 \frac{M_{B H}}{M_{\text {stor }}}\right)^{1 / 3}
$$

And also we have seen that, $D=10 R_{s^{\prime}}$, where the Schwarzschild radius is given by $r_{s}=\frac{2 M G}{c^{2}}$. Making use of these facts in the above expression, we can determine the mass of the black hole, for which the distance of tidal break up is about ten times the Schwarschild radius.

$$
M_{B H}=\left[\left(\frac{2 O G}{R_{W O} C^{2}}\right)^{3} \frac{M_{W D}}{4}\right]^{-1 / 2}
$$

This works out to about, $10^{33} \mathrm{~kg} \approx 10^{3} \mathrm{M}_{\Theta}$. That is, for a white dwart to be broken up due to the tidal force of a black hole, then the mass of the black hole should be of the above-mentioned mass.

The energy released during the break up corresponds to the gravitational binding energy given by,

$$
B . E=\frac{3}{5} \frac{G M_{W D}^{2}}{R_{W D}} \approx 10^{44} \mathrm{~J}
$$

A similar calculation can be done in the case of neutron star also. In the case of a neutron star, the neutron degeneracy pressure balances the gravitational pressure.

Equating the gravitational and the neutron degeneracy pressures we can calculate the radius of the neutron star and it is given by, $R_{N S}=\frac{\hbar^{2} M^{-\gamma / 3}}{G m_{n} m_{P}^{5 / 3}}$ 
For a neutron star of the mass of $1.5 \mathrm{M}_{\theta}$ the radius is about, $10 \mathrm{~km}$. The mass of the black hole to break up the neutron star due to the tidal force can be determined in the same way as before, that is, $M_{B H}=\left[\left(\frac{20 G}{R_{N S} \mathrm{c}^{2}}\right)^{3} \frac{M_{N S}}{4}\right]^{-1 / 2}$

This works out to about $10 M_{\theta}$. That is, a 10 solar mass black hole is required for the tidal break up of a neutron star.

The energy released in this case is given by,

$$
B . E=\frac{3}{5} \frac{G M_{N S}^{2}}{R_{N S}} \approx 10^{47} \mathrm{~J}
$$

\section{Conclusion}

The amount of energy released during the tidal break up of compact objects is very large. We have seen the effect of the fidal force in the vicinity of the black hole. We have also determined the energy released during such break ups in the case of normal stars as well as for compact objects. We have also shown that a super massive black hole cannot force the tidal break up of compact objects and from this we have arrived at the masses of the black holes required for the tidal break up of such compact objects.

\section{Bibliography}

1. Harry L Shipman, Black Holes, Quasars and the Universe, Houghton Mifflin and Company (1976)

2. William J Kaufmann III, Black Holes and Warped Space-time, W H Freeman and Co. (1979)

3. I Norliker, Violent Phenomena in the Universe, Oxford Univ. Press (1982)

4. Iian Nicholson, Grovity, Black Holes and the Universe, David and Charles Publication (1981)

5. http://cosmology.berkeley.edu

6. http://archive.ncsa.viuc.edu/Cyberia/NumRel/BlackHoles 